Editorial

\title{
Artificial Intelligence in Interventional Radiology
}

\author{
Shyamkumar N. Keshava ${ }^{1}$ Sanjeeva Kalva² \\ ${ }^{1}$ Department of Radiology, Christian Medical College, Vellore, \\ Tamil Nadu, India \\ 2Division of Interventional Radiology, Department of Radiology, \\ Massachusetts General Hospital, Boston, Massachusetts, \\ United States
}

J Clin Interv Radiol ISVIR 2019;3:71

Artificial Intelligence (AI) is an upcoming and revolutionary technology in many fields including medicine. AI in interventional radiology (IR) has been shown to be valuable in diagnosis and predicting outcomes following image-guided therapies. Abajian et al recently reported that outcomes following transarterial chemoembolization for patients with hepatocellular carcinoma may be predicted with the use of AI and machine learning techniques. ${ }^{1}$ Their methodology combined clinical data of patients and preprocedural magnetic resonance imaging. The relative performance of deeplearning neural network for detecting intracranial aneurysms using three-dimensional TOF-MRA as compared with the radiologists was recently reported. ${ }^{2}$ The role of machine learning in acute stroke has also been highlighted in many studies. ${ }^{3}$ In addition, there have been several publications regarding the role of $\mathrm{AI}$ in diagnostic imaging. ${ }^{4}$ These reports suggest that the role of AI is not limited to just process automation but also includes cognitive insight that is so far limited to the human brain.

The potential applications of AI in radiology include the ability to provide rapid diagnosis especially in the areas where diagnostic radiologists are not routinely available and improve the confidence in the diagnosis (similar to computer-aided diagnosis in mammography). The ability to incorporate clinical information, radiomics and patient's genetic information may prove AI-aided diagnosis more accurate. Such algorithmic approach could potentially play a role in triaging patients for IR and subsequent therapy by assessing the risks and making predictions about therapeutic outcomes. For instance, if the probability of a breast lesion being malignant is high, the patient may be considered for an image-guided biopsy. Similarly, in conditions such as stroke, where "time is money," AI could not only have a role in rapid diagnosis but facilitate prompt and effective therapeutic recommendations. Rapid interpretation of the extent of the penumbra, the potentially salvageable brain, could help the interventional radiologist decide whether the patient requires mechanical thrombectomy. Similarly, in cases of pulmonary embolism, AI may help in assessing the "cut off" and "reopened" branches quickly and assist in therapeutic decision making. Thus, AI in IR would help in optimally utilizing the manpower and resources. We can also anticipate AI helping IR in detecting early complications following IR procedures.

The AI techniques are still evolving. The ethical dilemmas in designing the AI algorithms would need to be discussed and agreed upon by the leaders of the world. More investment in research and validation of AI algorithms is needed. The other challenges include its availability, expertise, and economics. While there is a possibility of AI replacing some of the manpower, it is likely that AI would improve the overall outcomes of patient care by augmenting the physician performance.

\section{Conflict of Interest}

None declared.

\section{References}

1 Abajian A, Murali N, Savic LJ, et al. Predicting treatment response to intra-arterial therapies for hepatocellular carcinoma with the use of supervised machine learning-an artificial intelligence concept. J Vasc Interv Radiol 2018;29(6):850-857.e1

2 Faron A, Sichtermann T, Teichert N, et al. Performance of a deep-learning neural network to detect intracranial aneurysms from 3D TOF-MRA compared to human readers. Clin Neuroradiol 2019 (e-pub ahead of print) doi:10.1007/ s00062-019-00809-w.

3 Kamal H, Lopez V, Sheth SA. Machine learning in acute ischemic stroke neuroimaging. Front Neurol 2018;9:945 10.3389/ fneur.2018.00945

4 Choy G, Khalilzadeh O, Michalski M, et al. Radiology. Radiology 2018;288(2):318-328 shyam@cmcvellore.ac.in).
DOI https://doi.org/ 10.1055/s-0039-1694309 ISSN 2457-0214.
C2019 by Indian Society of Vascular and Interventional Radiology

\section{License terms}

$($ () (1) $\Theta \circledast$ 